\title{
SUBSTRUCTURE IN RICH CLUSTERS
}

\author{
Rachel Webster \\ Department of Astronomy, University of Toronto. \\ Michael Fitchett \\ CITA, University of Toronto. \\ Paul Hewett \\ Institute of Astronomy, Cambridge. \\ Matthew Colless \\ Department of Physics, Durham University.
}

We have developed a new statistical method based on maximum likelihood to test for the existence of two subclumps in data of arbitrary dimensionality (Fitchett 1987). The statistic, which is called the Lee function, can be calibrated using Monte Carlo simulations under various null hypotheses.

We have used this method to test for the existence of substructure in the central region of Coma (Fitchett and Webster 1987). We have analysed the 3-D data of Kent and Gunn (1982), and the 2-D data of Godwin and Peach (1977). The 2-D data is shown to have two subgroups at a statistically significant level. Two-body models with radial and circular orbits are calculated using virial determinations of the mass. Although the analysis has assumed that only two subclumps are present, the real distribution is probably more complex. If this analysis withstands the acquistion of new data, the central region of Coma is not relaxed.

The statistic has also been applied to the Cambridge sample of rich clusters (Colless and Hewett 1987). We have $\sim 40$ radial velocities for 14 rich southern clusters. About $35 \%$ of the clusters show substructure in the 2-D plots which is due to field galaxies. Five of the 14 clusters have significantly more field galaxies in the central region than would be predicted from the galaxy field counts. Application of the Lee statistic to the cluster galaxies suggests that $\sim 5$ of the clusters show evidence for substructure in the central regions. Dynamical models of the clusters have also been calculated.

\section{REFERENCES}

Colless, M.M. and Hewett, P.C. 1987, Mon. Not. R. astr. Soc., 224, 453.

Fitchett, M.J. 1987, Mon. Not. R. astr. Soc., , in press.

Fitchett, M.J. and Webster, R.L. 1987, Astrophys. J., 317, 653.

Godwin, J.C. and Peach, J.V. 1977, Mon. Not. R. astr. Soc., 181, 323.

Kent, S.M. and Gunn, J.E. 1982, Astron. J., 87, 945.

J. Audouze et al. (eds.), Large Scale Structures of the Universe, 537.

(c) 1988 by the IAU. 